\title{
Conformal Alpha Shapes
}

\author{
Frederic Cazals ${ }^{1} \quad$ Joachim Giesen $^{2 \dagger} \quad$ Mark Pauly $^{2} \quad$ Afra Zomorodian $^{3 \ddagger}$ \\ ${ }^{1}$ INRIA, BP93, 06902 Sophia Antipolis, France \\ ${ }^{2}$ Department of Computer Science, ETH Zurich, CH-8092 Zurich, Switzerland \\ ${ }^{3}$ Department of Computer Science, Stanford University, Stanford CA 94305, USA
}

\begin{abstract}
We define a new filtration of the Delaunay triangulation of a finite set of points in $\mathbb{R}^{d}$, similar to the alpha shape filtration. The new filtration is parameterized by a local scale parameter instead of the global scale parameter in alpha shapes. Since our approach shares many properties with the alpha shape filtration and the local scale parameter conforms to the local geometry we call it conformal alpha shape filtration. The local scale parameter is motivated from applications and previous algorithms in surface reconstruction. We show how conformal alpha shapes can be used for surface reconstruction of non-uniformly sampled surfaces, which is not possible with alpha shapes.
\end{abstract}

Categories and Subject Descriptors (according to ACM CCS): I.3.5 [Computer Graphics]: Curve, surface, solid, and object representations

\section{Introduction}

The method of alpha shapes was originally motivated for the study of points in the plane [EKS83]. This method was later generalized to higher dimensions and weighted points [EM94]. Alpha shapes define a family of simplicial complexes parameterized by $\alpha \in \mathbb{R}$. These $\alpha$-complexes have vertices in the point set and simplices from the points' Delaunay triangulation. Consequently, alpha complexes are efficiently computable. The family implies a filtration, a partial ordering of the simplices of the Delaunay triangulation, that may be used for multi-scale topological analysis of the point cloud. It is this rich structure that makes alpha shapes popular in many applications ranging from bio-geometric modeling [EFL98], where atoms are modeled as weighted points, to surface reconstruction, where the surface of some solid is sampled.

Alpha shapes have influenced the development of provable surface reconstruction algorithms in computational geometry. By "provable", we mean geometric and topological

\footnotetext{
$\dagger$ Partially supported by the Swiss National Science Foundation under the project "Non-linear manifold learning".

$\ddagger$ Partially supported by DARPA under grant 32905 .
}

guarantees that are based on assumptions on the sampling. We distinguish two major lines of Delaunay-based surface reconstruction algorithms. The first line considers filtering the Delaunay triangulation of a point cloud. Alpha shapes is one such filter as each $\alpha$-complex specifies a subset of the simplices. Beginning with the seminal work of Amenta and Bern, there have been a flurry of such algorithms proposed, the most significant of which are the Crust and the Cocone algorithms [AB99, ACDL00]. The second line of research takes the fundamentally different approach of examining the critical points of a discrete or continuous flow based on the Delaunay complex [Ede04, GJ03]. These critical points are related to the critical $\alpha$-complex simplices: the simplices at which the complex undergoes a topological change.

Although alpha shapes have inspired fruitful research on surface reconstruction, the method's utility is limited. First, alpha shapes define a family of complexes, but it is not clear which $\alpha$-complex is suitable for reconstruction. Second, the chosen $\alpha$ fixes a global scale, so the method can be successful only for uniform sampling. The algorithms that have been successful in practice all use local filters to cope with nonuniform sampling.

In this paper, we introduce conformal alpha shapes which use a local scale parameter $\hat{\alpha}$ instead of the global scale pa- 
rameter $\alpha$. We show that conformal alpha shapes share many properties with ordinary alpha shapes, but have additional properties that are useful for surface reconstruction. In the rest of the paper, we study the geometric and topological consequences of localizing the scale parameter within the framework of surface reconstruction. We begin by defining conformal alpha shapes in Section 2. In Section 3, we describe the geometric consequences of utilizing conformal alpha shapes. In Section 4, we consider the topological repercussions.

\section{Conformal Alpha Shapes}

In this section, we begin by briefly describing the background necessary for our work, including the definition of alpha complexes as a family of subcomplexes of the Delaunay triangulation. We then introduce conformal alpha shapes and prove that it also provides a family of subcomplexes as the prior method.

\subsection{Preliminaries}

A point set $P \subset \mathbb{R}^{d}$ is in general position if there are no $k \leq d+1$ points on a common $(k-2)$-flat or $k \leq d+2$ points on a common $(k-3)$-sphere. In the following, we always assume general position as this assumption simplifies the exposition and is justified in practice [Ede01]. A $k$-simplex $\sigma$ is the convex hull of $k+1$ points $S \subseteq P$. A simplex $\tau$ defined by $T \subseteq S$ is a face of $\sigma$ and $\sigma$ is a co-face of $\tau$. A simplicial complex $K$ is a finite set of simplices that meet along faces, all of which are in $K$. A filtration of a complex $K$ is a nested subsequence of complexes $\emptyset=K^{0} \subseteq K^{1} \subseteq \ldots \subseteq K^{m}=K$.

The Voronoï diagram $V(P)$ of $P$ is a cell decomposition of $\mathbb{R}^{d}$ into convex polyhedra. Every Voronoï cell $V_{p}$ corresponds to exactly one sample point $p \in P$ and contains all points of $\mathbb{R}^{d}$ closest to $p$. That is,

$$
V_{p}=\left\{x \in \mathbb{R}^{d} \mid\|x-p\| \leq\|x-q\|, \forall q \in P\right\} .
$$

Closed facets shared by $d-k+1$ Voronoï cells are called Voronoï $k$-facets.

The Delaunay triangulation $D(P)$ of $P$ is the dual of the Voronoï diagram. Whenever a collection $V_{p_{1}}, \ldots, V_{p_{k}}$ of Voronoï cells have a non-empty intersection, the simplex defined on the corresponding points $p_{1}, \ldots, p_{k}$ is in $D(P)$. The Delaunay triangulation is a simplicial complex that decomposes the convex hull of the points in $P$. In the rest of the paper, all simplices will be Delaunay. At times, we will restate this to remind the reader.

\subsection{Alpha Shapes}

For a given value of $\alpha \in[0, \infty)$, alpha balls are balls of radius $\alpha$ around the points in $P$. The corresponding alpha complex of $P$ is the Delaunay triangulation of $P$ restricted to the alpha balls. A simplex belongs to the alpha complex if the
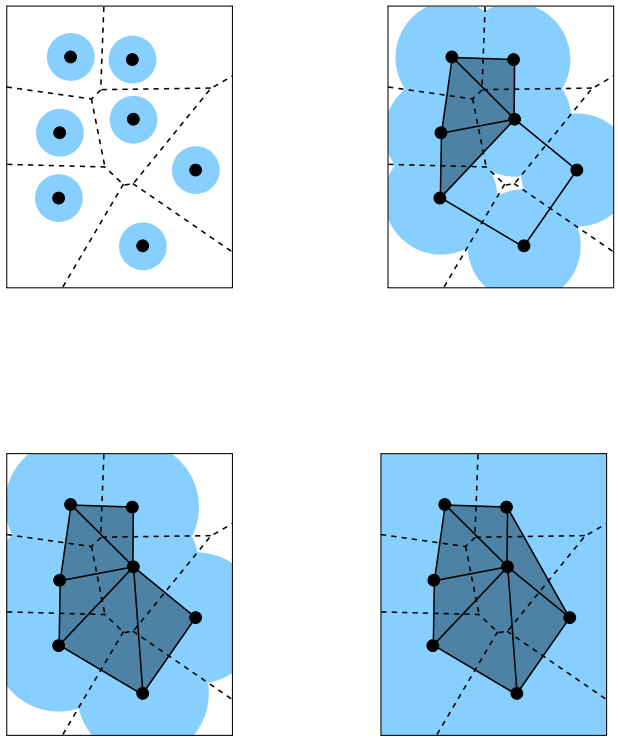

Figure 1: Alpha shapes for growing values of $\alpha$.

Voronoï cells of its vertices have a common non-empty intersection with the set of alpha balls. Note that at $\alpha=0$, the alpha complex consists just of the set $P$, and for sufficiently large $\alpha$, the alpha complex is the Delaunay triangulation $D(P)$ of $P$. For any simplex $\sigma \in D(P)$, let $\alpha(\sigma)$ be the $\alpha$ value at which $\sigma$ appears for the first time in the alpha complex. The alpha shape filtration is the sequence of alpha complexes obtained from growing $\alpha$ from zero to infinity. We show a few complexes from the alpha shape filtration for a small set of points in Figure 1.

\subsection{Definition}

For $p \in P$, let $D_{p} \subseteq D(P)$ denote the simplices incident on $p$. The alpha values determine a partial ordering on $D_{p}$, one which we make into a total ordering by sorting according to dimension and breaking the remaining ties arbitrarily. We may then view $D_{p}$ as a sequence of simplices with nondecreasing alpha values $\alpha_{p}^{1} \leq \cdots \leq \alpha_{p}^{n}$. Note that $\alpha_{p}^{1}=0$ since the first simplex in $D_{p}$ is the point $p$ which appears at $\alpha=0$. Let $\alpha_{p}^{-}<\alpha_{p}^{+}$be two $\alpha$ values in $\left\{\alpha_{p}^{i}\right\}_{i}$. We will specify how to choose these values later in the paper. We now rescale $\alpha_{p}^{i}$ using these local values:

$$
\hat{\alpha}_{p}^{i}=\frac{\alpha_{p}^{i}-\alpha_{p}^{-}}{\alpha_{p}^{+}} .
$$

We call $\hat{\alpha}_{p}^{i}$ the internal alpha scale. This scale is invariant to Euclidean transformations and scaling, so it is conformal. 
As in alpha shapes, we consider a restricted Delaunay triangulation for each value $\hat{\alpha} \in(-\infty, \infty)$. But the restriction is not to the set of alpha balls, but a new set of balls whose radii are determined from their internal alpha scales. We put a ball of radius $\alpha_{p}$ at each point $p \in P$, where

$$
\alpha_{p}(\hat{\alpha})=\alpha_{p}^{+} \hat{\alpha}+\alpha_{p}^{-},
$$

and a ball of negative radius is defined to be empty. Let $C_{p}^{\hat{\alpha}}$ be the intersection of the Voronoï cell $V_{p}$ and the ball at $p$ and let $C^{\hat{\alpha}}$ be the interior of $\cup_{p \in P} C_{p}^{\hat{\alpha}}$. The conformal alpha shape (complex) is the Delaunay triangulation of $P$ restricted to $C^{\hat{\alpha}}$.

As in the definition of $\alpha(\sigma)$, let $\hat{\alpha}(\sigma)$ be the $\hat{\alpha}$ value at which $\sigma$ appears for the first time in the conformal alpha shape. We may compute the $\hat{\alpha}(\sigma)$ from the value of $\alpha(\sigma)$. Let $p_{1}, \ldots, p_{k} \in P$ be the vertices of $\sigma$. Then,

$$
\hat{\alpha}(\sigma)=\max _{1 \leq i \leq k} \inf \left\{\hat{\alpha} \mid \alpha_{p_{i}}(\hat{\alpha}) \geq \alpha(\sigma)\right\} .
$$

Lemma 1 The sequence of conformal alpha shapes obtained by growing $\hat{\alpha}$ from zero to infinity is a filtration of the Delaunay triangulation $D(P)$ of $P$.

Proof We need to show the following: (1) If $\hat{\alpha}<\hat{\alpha}^{\prime}$, the simplices in the conformal $\hat{\alpha}$-shape are also in the conformal $\hat{\alpha}^{\prime}$-shape. (2) For sufficiently large $\hat{\alpha}$, the conformal $\hat{\alpha}$-shape is $D(P)$. (3) A simplex $\sigma \in D(P)$ is earlier than all its cofaces $\tau$ in the filtration, that is, $\hat{\alpha}(\sigma) \leq \hat{\alpha}(\tau)$.

Property (1) holds as $C^{\hat{\alpha}} \subset C^{\hat{\alpha}^{\prime}}$ for $\hat{\alpha}<\hat{\alpha}^{\prime}$. Property (2) holds as $C^{\hat{\alpha}}$ covers $\mathbb{R}^{d}$ in the limit as $\hat{\alpha}$ approaches infinity. For property (3), let $S=\left\{p_{i}\right\}_{i}$ be the vertices of $\sigma$. The co-face $\tau$ also has $S$ as vertices, along with some additional vertices. There exists a point $p_{i} \in S$ such that

$$
\alpha_{p_{i}}(\hat{\alpha}(\sigma))=\alpha(\sigma) \leq \alpha(\tau) \leq \alpha_{p_{i}}(\hat{\alpha}(\tau)),
$$

which implies $\hat{\alpha}(\sigma) \leq \hat{\alpha}(\tau)$ as $\alpha_{p_{i}}$ is a monotonically increasing function.

\section{Geometry}

We wish to use conformal alpha shapes to reconstruct a smooth surface $S$ in $\mathbb{R}^{3}$ from a finite sampling $P$. In this section, we describe a geometrical approach very much in line with the philosophy behind the Crust and Cocone algorithms. We begin with the common geometric definitions and then examine the geometry of the reconstruction.

\subsection{Preliminaries}

Suppose we are given a smooth surface $S$ embedded in $\mathbb{R}^{3}$. An open ball is empty if it does not contain any point from $S$. An empty ball is maximal if it is not contained in a larger empty ball. The medial axis $M(S)$ of $S$ is the union of the centers of all maximal open balls. The distance of a point $x \in S$ to the medial axis is $M(S)$ its local feature size. We define $f: S \rightarrow \mathbb{R}$,

$$
f(x)=\inf _{y \in M(S)}\|x-y\|,
$$

to be the function that assigns the local feature size to a point.

An $\varepsilon$-sample of $S$ is a subset $P \subseteq S$ such that every point $x \in S$ has a point $p \in P$ at distance at most $\varepsilon f(x)$. An $\varepsilon$ sampling is uniform if every point has a point in $P$ at distance at $\operatorname{most}_{\inf } \in S f(x)$. Although the sampling density may vary non-uniformly across $S$, the density is bounded below by the smallest feature size. For sufficiently small $\varepsilon$, every $\varepsilon$-sample is uniform but it depends on $S$ what sufficiently small means.

Let $V_{p}$ be the Voronoï cell of a sample point $p \in P$. If $V_{p}$ is bounded, we let $\vec{u}$ be the vector from $p$ to the Voronoi vertex in $V_{p}$ that has the largest distance to $p$. Otherwise, $V_{p}$ is unbounded and we let $\vec{u}$ be a vector in the average direction of all unbounded Voronoï edges incident to $V_{p}$. The pole of $V_{p}$ is the Voronoï vertex $p^{*}$ in $V_{p}$ with the largest distance to $p$ such that the vector $\vec{u}$ and the vector from $p$ to $p^{*}$ make an angle larger than $\pi / 2$ [AB99].

\subsection{Reconstruction}

We begin by specifying the internal alpha scale parameters $\alpha_{p}^{-}$and $\alpha_{p}^{+}$for a sample point $p \in P$. Let $\alpha_{p}^{-}=\alpha_{p}^{1}=0$. Let $\alpha_{p}^{+}$be the $\alpha$ value at which the simplex dual to the pole $p^{*}$ appears in the ordinary alpha shape, that is, $\alpha_{p}^{+}=\left\|p-p^{*}\right\|$. Note that with these values for the parameters, the points in $P$ all appear at $\hat{\alpha}=0$. This implies that all the simplices appear at non-negative $\hat{\alpha}$ values.

The restricted Voronoï diagram $V_{S}(P)$ is the Voronoï diagram $V(P)$ intersected with the surface $S$. The restricted Delaunay triangulation $D_{S}(P)$ is its dual and is necessarily a subset of the Delaunay triangulation. In the rest of the section, we use $\eta=\varepsilon /(1-\varepsilon)$ for notational brevity. We begin with the following result.

Lemma 2 ([AB99]) Let $P$ be an $\varepsilon$-sample of a smooth surface $S$ and let $p \in P$ be a sample point. Then,

- For any point $x$ in the cell $V_{p}$ in $V_{S}(P),\|p-x\| \leq \eta f(p)$.

- For any point $x$ in the intersection of $V_{p}$ with the hyperplane containing $p$ and orthogonal to $p^{*}-p$,

$$
\|x-p\| \leq \frac{\eta f(p)}{\sin \left(\frac{\pi}{2}-3 \arcsin \eta\right)} .
$$

Lemma 3 Let $P$ be an $\varepsilon$-sample of a smooth surface $S$. Then, all conformal alpha shapes for $\hat{\alpha} \geq \eta$ contain $D_{S}(P)$.

Proof For $p \in P$, let $\alpha_{p}^{i}$ be the largest $\alpha$ value at which a simplex from $D_{S}(P)$ incident to $p$ appears in the ordinary alpha shape. By Lemma 2 we have that

$$
\alpha_{p}^{i} \leq \eta f(p) .
$$


We also have $\alpha_{p}^{+} \geq f(p)$ by our choice of $\alpha_{p}^{+}$. Therefore,

$$
\hat{\alpha}_{p}^{i}=\frac{\alpha_{p}^{i}}{\alpha_{p}^{+}} \leq \frac{\eta f(p)}{\alpha_{p}^{+}} \leq \eta .
$$

This implies the statement of the lemma.

Essentially, Lemma 3 asserts that the alpha shape for a large enough $\hat{\alpha}$ contains certain simplices of the Delaunay triangulation of a surface sampling. The following lemma states that the conformal alpha shape does not contain simplices that are too large. The idea behind this lemma is that the Voronoï cells of the sample points are long and thin and directed almost along the normals at the sample points. Therefore, edges that are almost tangential to the surface will appear early in the conformal alpha shape.

Lemma 4 Let $P$ be an $\varepsilon$-sample of a smooth surface $S$. The neighbors of $p \in P$ in a conformal alpha shape for small values of $\hat{\alpha}$ are at distance at most

$$
\left(\frac{1+\hat{\alpha}}{1-\hat{\alpha}}\right)\left(\frac{2 \eta}{\sin \left(\frac{\pi}{2}-3 \arcsin \eta\right)}\right) f(p) .
$$

Proof Let $l$ be the vector $p^{*}-p$ as in Figure 2 to the left. We first want to bound the width of smallest cylinder with axis $l$ that contains the intersection of the Voronoï cell $V_{p}$ of $p$ with the ball of radius $\alpha_{p}$ centered at $p$. Let $x$ be any point in the intersection of the boundary of $V_{p}$ and the hyperplane containing $p$ and orthogonal to $l$. Any hyperplane $H$ supporting $V_{p}$ at $x$ must have $p$ and $p^{*}$ on the same side. In the limiting case, $p^{*}$ is contained in $H$ and so we consider this case. Then $H$ contains a line $l^{\prime}$ through $p^{*}$ and $x$. Let $\beta$ be the acute angle made by $l$ and $l^{\prime}$ at $p^{*}$, shown in Figure 2

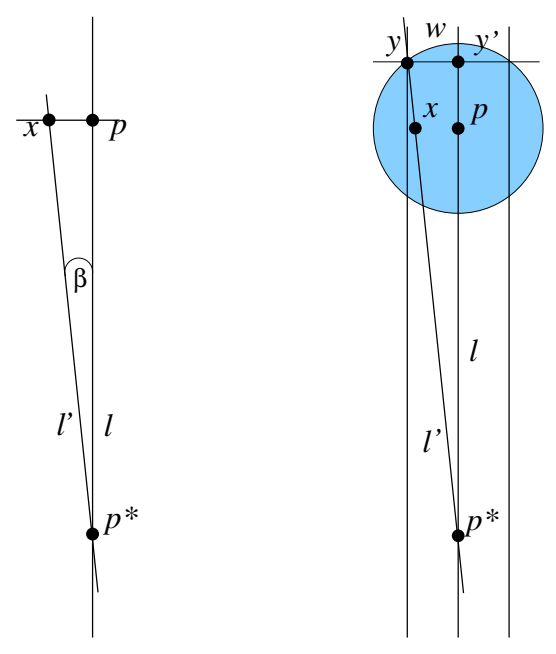

Figure 2: Bounding $\tan \beta$ and the width $w$ of the cylinder.
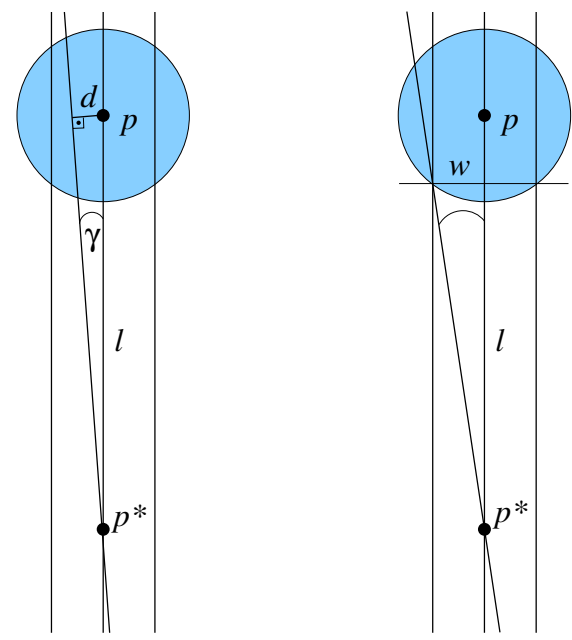

Figure 3: Bounding $\tan \gamma$ and the distance $d$ to a neighbor in the conformal alpha shape.

to the left. By Lemma 2,

$$
\tan \beta=\frac{\|x-p\|}{\left\|p-p^{*}\right\|} \leq \frac{\eta f(p)}{\alpha_{p}^{+} \sin \left(\frac{\pi}{2}-3 \arcsin \eta\right)} .
$$

The line $l^{\prime}$ intersects the boundary of the ball of radius $\alpha_{p}(\hat{\alpha})$ centered at $p$ in at most two points, as shown in Figure 2 to the right. Let $y$ be the intersection point furthest from $p^{*}$. Since we haven chosen $p^{*}$ and $H$ to be the limiting case, the distance of $y$ to $l$ is an upper bound for the width $w$ of the cylinder we are looking for. Let $y^{\prime}$ be the projection of $y$ onto $l$. Then,

$$
\begin{aligned}
w & \leq\left\|y^{\prime}-p^{*}\right\| \tan \beta \\
& =\left(\left\|p-p^{*}\right\|+\left\|y^{\prime}-p\right\|\right) \tan \beta \\
& \leq\left(\alpha_{p}^{+}+\alpha_{p}(\hat{\alpha})\right) \tan \beta .
\end{aligned}
$$

Now let $F$ be the affine hull of a Voronoï facet in $V_{p}$ that is intersected by $C_{p}^{\hat{\alpha}}$. Again, $p^{*}$ must be on the same side of $F$ as $p$. The line $l$ intersects $F$ in a unique point. Let $\gamma$ be the minimum angle between $l$ and $F$ at this intersection point, as shown in Figure 3 to the right. Then, we have

$$
\tan \gamma \leq \frac{w}{\left\|p-p^{*}\right\|-\alpha_{p}(\hat{\alpha})}=\frac{w}{\alpha_{p}^{+}-\alpha_{p}(\hat{\alpha})} .
$$

The length $d$ of the Delaunay edge dual to the Voronoi facet corresponding to $F$ may be bounded by:

$$
\begin{aligned}
d & \leq 2\left\|p-p^{*}\right\| \sin \gamma \\
& =2 \alpha_{p}^{+} \sin \gamma \\
& \leq 2 \alpha_{p}^{+} \tan \gamma
\end{aligned}
$$




$$
\begin{aligned}
& \leq \frac{2 w \alpha_{p}^{+}}{\alpha_{p}^{+}-\alpha_{p}(\hat{\alpha})} \\
& =\left(\frac{\alpha_{p}^{+}+\alpha_{p}(\hat{\alpha})}{\alpha_{p}^{+}-\alpha_{p}(\hat{\alpha})}\right) 2\left\|p-p^{*}\right\| \tan \beta \\
& \leq\left(\frac{\alpha_{p}^{+}+\alpha_{p}(\hat{\alpha})}{\alpha_{p}^{+}-\alpha_{p}(\hat{\alpha})}\right)\left(\frac{2 \eta}{\sin \left(\frac{\pi}{2}-3 \arcsin \eta\right)}\right) f(p) .
\end{aligned}
$$

This implies that all neighbors of $p$ in a conformal alpha shape are at distance at most

$$
\begin{aligned}
\left(\frac{\alpha_{p}^{+}+\alpha_{p}^{+} \hat{\alpha}}{\alpha_{p}^{+}-\alpha_{p}^{+} \hat{\alpha}}\right) & \left(\frac{2 \eta}{\sin \left(\frac{\pi}{2}-3 \arcsin \eta\right)}\right) f(p) \\
= & \left(\frac{1+\hat{\alpha}}{1-\hat{\alpha}}\right)\left(\frac{2 \eta}{\sin \left(\frac{\pi}{2}-3 \arcsin \eta\right)}\right) f(p) .
\end{aligned}
$$

This completes the proof.

Basically, Lemma 4 states that the conformal alpha shape is contained in a thickening of the surface $S$ where the thickening factor with respect to the feature size depends on $\hat{\alpha}$ and $\eta$ via

$$
\left(\frac{1+\hat{\alpha}}{1-\hat{\alpha}}\right)\left(\frac{2 \eta}{\sin \left(\frac{\pi}{2}-3 \arcsin \eta\right)}\right) .
$$

Note that the thickening factor has two terms: a first part that only depends on the scale parameter $\hat{\alpha}$, and a second part that only depends on the sampling density $\varepsilon$. If $\hat{\alpha}=\eta$ and $\varepsilon<0.1$, then $\eta<0.112$ and this factor is less than 1 . That is, the conformal alpha shape of an $\varepsilon$-sample with $\varepsilon<0.1$ does not contain any point from the medial axis of the surface. This is true regardless of what the surface is provided it is smooth. This contrasts with ordinary alpha shapes where for any $\varepsilon>0$, we can give a surface such that the alpha shape contains a point of the medial axis.

The Crust and Cocone algorithms begin by filtering a set of candidate triangles from the Delaunay triangulation. An edge is sharp if it has either a single incident triangle, or if any two consecutive triangles incident to it form an angle more than $3 \pi / 2$. In the second step, the algorithms remove all triangles incident on sharp edges. Finally, the algorithms compute a reconstruction by "walking" on either the inside or outside of the remaining set of candidate triangles. The resulting surface is homeomorphic to the original surface $S$ if $P$ is a sufficiently dense $\varepsilon$-sample. The homeomorphism proof needs two properties of the set of candidate triangles. First, it has to contain all triangles of the restricted Delaunay triangulation $D_{S}(P)$. Second, all triangles need to have a small circumradius compared to the feature size at their vertices.

We now show that a conformal alpha shape for a suitable value of $\hat{\alpha}$ may be used as the source of the candidate triangles, still giving us the topological guarantees after prun- ing and walking. Suppose we are given an $\varepsilon$-sample $P$ of a smooth closed surface $S$ with $\varepsilon<0.1$. To show the homeomorphism property, we need to satisfy the two requirements discussed above. By Lemma 3, we know that the conformal alpha shape for $\hat{\alpha}=\eta=\varepsilon /(1-\varepsilon)$ contains the restricted Delaunay triangulation $D_{S}(P)$. It remains to show that all triangles in this conformal alpha shape have a small circumradius.

Lemma 5 Let $P$ be an $\varepsilon$-sample of a smooth surface $S$. All triangles incident to $p \in P$ in a conformal alpha shape for $\hat{\alpha}<1$ have circumradius of at most

$$
\left(\frac{1+\hat{\alpha}}{1-\hat{\alpha}}\right)\left(\frac{\eta}{\sin \left(\frac{\pi}{2}-3 \arcsin \eta\right)}\right) f(p) .
$$

The proof is basically the same as for Lemma 4. Therefore, we may compute a homeomorphic reconstruction of $S$ from the conformal alpha shape of $P$ with $\hat{\alpha}=\eta$.

\section{Topology}

In this section, we study both the ordinary and the conformal alpha shape filtrations. A filtration allows us to track of topological changes at different scales. Here, the scale parameter is either $\alpha$ or $\hat{\alpha}$. The topology of the respective alpha shapes changes only at a finite number of critical $\alpha$ values as both have a finite number of simplices. We characterize these values and uncover the relationship between the critical values of conformal alpha shapes and those of the ordinary alpha shapes.

A simplex $\sigma$ is $\alpha$-late if $\alpha(\rho)<\alpha(\sigma)$ for all faces $\rho$, and $\alpha$-early if $\alpha(\sigma)<\alpha(\tau)$ for all co-faces $\tau$. If $\sigma$ is both $\alpha$ late and $\alpha$-early, it is $\alpha$-critical. We similarly have $\hat{\alpha}$-late, $\hat{\alpha}$-early, and $\hat{\alpha}$-critical. We define every vertex to be $\alpha$ - and $\hat{\alpha}$-late and every $d$-dimensional simplex to be $\alpha$ - and $\hat{\alpha}$-early. Note that by the filtration property we always have $\alpha(\rho) \leq$ $\alpha(\sigma) \leq \alpha(\tau)$ and $\hat{\alpha}(\rho) \leq \hat{\alpha}(\sigma) \leq \hat{\alpha}(\tau)$ for faces $\rho$ and cofaces $\tau$ of simplex $\sigma$.

Lemma 6 The homotopy type of the alpha shape (ordinary or conformal) of a finite point set in general position changes only when a critical simplex enters the shape.

Proof Clearly, the homotopy type of an alpha shape (ordinary or conformal) changes when a critical simplex appears. If a $k$-dimensional critical simplex appears either the $(k-1)$ th Betti number of the alpha shape decreases by 1 or the $k$ th Betti number increases by 1 in simplicial homology. At the appearance of 0-dimensional simplices (vertices), the 0th Betti number always increases, and at the appearance of critical $d$-dimensional simplices, the $(d-1)$-th Betti number always decreases.

In the following, we restrict our exposition to $\hat{\alpha}(\cdot)$, but all arguments also hold for $\alpha(\cdot)$. We need to show that the homotopy type of the alpha shape does not change for non-critical simplices. If a $k$-dimensional simplex $\sigma$ is non-critical, then either $\sigma$ has a $(k-1)$-dimensional face $\rho$ with $\hat{\alpha}(\rho)=\hat{\alpha}(\sigma)$ 
or $\sigma$ is the face of a $(k+1)$-dimensional simplex with $\hat{\alpha}(\sigma)=$ $\hat{\alpha}(\tau)$. Note that if $\hat{\alpha}(\rho)=\hat{\alpha}(\sigma)$, then $\rho$ is non-critical and if $\hat{\alpha}(\sigma)=\hat{\alpha}(\tau)$, then $\tau$ is non-critical.

Let $\sigma$ be the highest dimensional simplex involved in a noncritical alpha event, that is, the highest-dimensional simplex among those that appear at the same $\hat{\alpha}$ value. The dimension $k$ of $\sigma$ is at least two as a non-critical event may not involve just vertices and edges: if an edge is early, it has to be critical as by definition it is always late. By our assumption, $\sigma$ cannot be early, so it must have at least one $(k-1)$-dimensional face $\rho$ with $\hat{\alpha}(\rho)=\hat{\alpha}(\sigma)$. If we can show that there is exactly one such face $\rho$ then we are done as there is a straightforward deformation retraction of $\sigma$ to $\partial \sigma \backslash \rho$ and the homotopy type of the alpha shape does not change.

We need to show that there is only one $(k-1)$-dimensional face $\rho$ of $\sigma$ with $\hat{\alpha}(\rho)=\hat{\alpha}(\sigma)$. Let $p_{1}, \ldots, p_{k+1} \in P$ be the vertices of $\sigma$. The $(k-1)$-dimensional faces $\rho_{i}$ of $\sigma$ are the convex hull of the vertex sets $\left\{p_{1}, \ldots, p_{k+1}\right\} \backslash\left\{p_{i}\right\}$ for $1 \leq$ $i \leq k$. Let $V$ be the Voronoï facet dual to $\sigma$ and $V_{i}$ be the Voronoï facet dual to $\rho_{i}$. As $\sigma$ is not $\hat{\alpha}$-late, there is a $V_{i}$ with

$$
\min \left\{\hat{\alpha} \mid C^{\hat{\alpha}} \cap V_{i} \neq \emptyset\right\}=\min \left\{\hat{\alpha} \mid C^{\hat{\alpha}} \cap V \neq \emptyset\right\},
$$

as $\hat{\alpha}\left(\rho_{i}\right)=\hat{\alpha}(\sigma)$. Now assume that there is another face $\rho_{j} \neq$ $\rho_{i}$ with the same property

$$
\min \left\{\hat{\alpha} \mid C^{\hat{\alpha}} \cap V_{j} \neq \emptyset\right\}=\min \left\{\hat{\alpha} \mid C^{\hat{\alpha}} \cap V \neq \emptyset\right\} .
$$

Then, we have

$$
C^{\hat{\alpha}(\sigma)} \cap V_{i}=C^{\hat{\alpha}(\sigma)} \cap V_{j}=C^{\hat{\alpha}(\sigma)} \cap V .
$$

The intersection $C^{\hat{\alpha}(\sigma)} \cap V$ must be a single point since $\hat{\alpha}(\sigma)$ is the smallest $\hat{\alpha}$ such that $C^{\hat{\alpha}} \cap V \neq \emptyset$. Let $x$ be the intersection point. Since $\hat{\alpha}\left(\rho_{i}\right)=\hat{\alpha}(\sigma)$, there must exist a vertex $q \in \rho_{i}$ with $\operatorname{argmin}_{y \in V_{i}}\|q-y\|=x$. But any point $y \in V_{i}$ has the same distance to all vertices of $\rho_{i}$. So, for all vertices $q \in \rho_{i}$,

$$
\underset{y \in V_{i}}{\operatorname{argmin}}\|q-y\|=x .
$$

Similarly, for all vertices $r \in \rho_{j}$,

$$
\underset{y \in V_{j}}{\operatorname{argmin}}\|r-y\|=x .
$$

Since $\sigma$ is at least two-dimensional, i.e., $k \geq 2, \rho_{i}$ and $\rho_{j}$ must have at least one vertex $p \in P$ in common. For this vertex $p$,

$$
\underset{y \in V_{i}}{\operatorname{argmin}}\|p-y\|=\underset{y \in V_{j}}{\operatorname{argmin}}\|p-y\|=x,
$$

and $x$ is the center of the circumcircle of the triangle $p_{i} p_{j} p$ :

$$
\left\|p_{i}-x\right\|=\left\|p_{j}-x\right\|=\|p-x\| .
$$

By construction, the line through $p$ and $p_{j}$ is orthogonal to the affine hull of $V_{i}$ as the line segment $p p_{j}$ is an edge of $\rho_{i}$. Similarly, the line through $p$ and $p_{i}$ is orthogonal to the affine hull of $V_{j}$. But this is impossible since the Voronoï cell $V_{p}$ is convex and we assumed the point set $P$ is in general

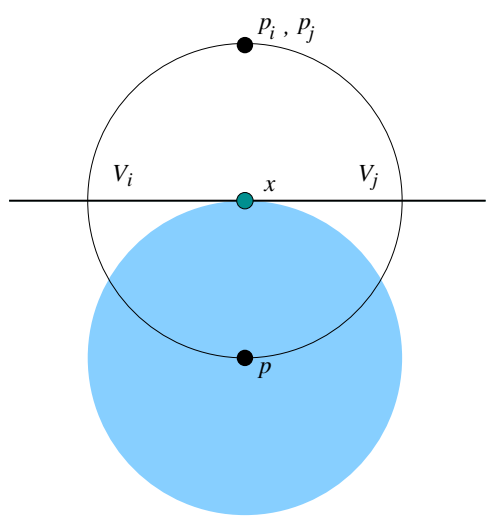

Figure 4: The only position of $p_{i}$ and $p_{j}$ is degenerate, taking the convexity of $V_{p}$ into account.

position, as shown in Figure 4. Therefore $p_{i}=p_{j}$, arriving at a contradiction. This completes the proof.

Lemma 7 Every $\alpha$-critical Delaunay simplex $\sigma$ is also $\hat{\alpha}$ critical.

Proof Let $p_{1}, \ldots, p_{k} \in P$ be the vertices of $\sigma$. We begin by showing that if $\sigma$ is $\alpha$-late, then it is also $\hat{\alpha}$-late. If $\sigma$ is $\alpha$ late, $\alpha\left(\rho_{i}\right)<\alpha(\sigma)$ for all its $(k-2)$-dimensional faces $\rho_{i}$, $1 \leq i \leq k$. So,

$$
\begin{aligned}
\hat{\alpha}\left(\rho_{i}\right) & =\max _{\substack{1 \leq j \leq k \\
j \neq i}} \inf \left\{\hat{\alpha} \mid \alpha_{p_{j}}(\hat{\alpha}) \geq \alpha\left(\rho_{j}\right)\right\} \\
& <\max _{\substack{1 \leq j \leq k \\
j \neq i}} \inf \left\{\hat{\alpha} \mid \alpha_{p_{j}}(\hat{\alpha}) \geq \alpha(\sigma)\right\} \\
& \leq \max _{1 \leq j \leq k} \inf \left\{\hat{\alpha} \mid \alpha_{p_{j}}(\hat{\alpha}) \geq \alpha(\sigma)\right\} \\
& =\hat{\alpha}(\sigma),
\end{aligned}
$$

for $1 \leq i \leq k$. That is, $\sigma$ is also $\hat{\alpha}$-late. We now show the reverse statement: if $\sigma$ is $\alpha$-early, then it is also $\hat{\alpha}$-early. Let $\tau$ be any $k$-dimensional co-face of $\sigma$ and let $p_{k+1}$ be be the additional vertex of $\tau$. Since $\sigma$ is $\alpha$-early, $\alpha(\sigma)<\alpha(\tau)$. We have

$$
\begin{aligned}
\hat{\alpha}(\sigma) & =\max _{1 \leq j \leq k} \inf \left\{\hat{\alpha} \mid \alpha_{p_{j}}(\hat{\alpha}) \geq \alpha(\sigma)\right\} \\
& <\max _{1 \leq j \leq k} \inf \left\{\hat{\alpha} \mid \alpha_{p_{j}}(\hat{\alpha}) \geq \alpha(\tau)\right\} \\
& \leq \max _{1 \leq j \leq k+1} \inf \left\{\hat{\alpha} \mid \alpha_{p_{j}}(\hat{\alpha}) \geq \alpha(\tau)\right\} \\
& =\hat{\alpha}(\tau) .
\end{aligned}
$$

Therefore, $\sigma$ is also $\hat{\alpha}$-early. Since it was $\hat{\alpha}$-late from before, $\sigma$ is $\hat{\alpha}$-critical.

Note that the reverse of Lemma 7 is not true in general. There can be more $\hat{\alpha}$-critical simplices then there are $\alpha$-critical ones, as demonstrated in Figure 5.

We get two permutations of the $\alpha$-critical simplices according to the $\alpha$ and $\hat{\alpha}$ values, respectively. The distance 


\section{F. Cazals et al. / Conformal Alpha Shapes}

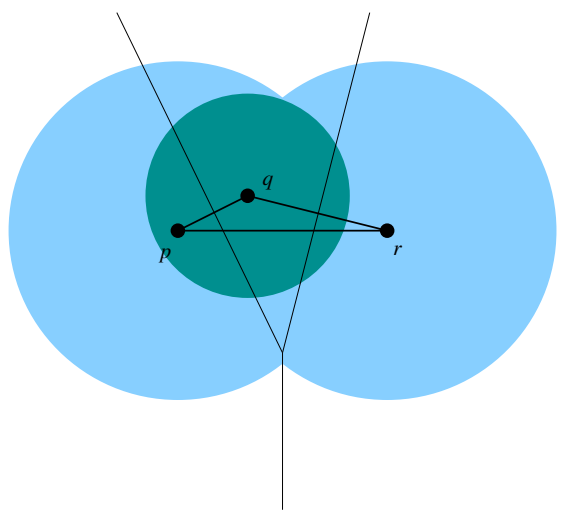

Figure 5: The reverse of Lemma 7 is not always true. Triangle pqr is $\hat{\alpha}$-critical but not $\alpha$-critical if we assume that the poles $p^{*}$ and $r^{*}$ are somewhere below (not shown in the figure) and the pole $q^{*}$ is the circumcenter of the triangle pqr.

of these permutations is a measure of the non-uniformity in data set $P$. Suitable measures of the distance of two permutations $\pi_{1}$ and $\pi_{2}$ include Spearman rules

$$
\begin{aligned}
F\left(\pi_{1}, \pi_{2}\right) & =\sum_{i}\left|\pi_{1}(i)-\pi_{2}(i)\right| \\
R^{2}\left(\pi_{1}, \pi_{2}\right) & =\sum_{i}\left|\pi_{1}(i)-\pi_{2}(i)\right|^{2},
\end{aligned}
$$

as well as Kendall's tau

$$
\begin{aligned}
K\left(\pi_{1}, \pi_{2}\right)= & \text { minimum number of bubble sort steps } \\
& \text { taking } \pi_{1}^{-1} \text { to } \pi_{2}^{-1} .
\end{aligned}
$$

The $\alpha$-critical simplices have a simple characterization.

Lemma 8 ([Ede04]) The $\alpha$-critical Delaunay simplices are exactly those that have a non-empty intersection with their dual Voronoï cells. This intersection is a unique point, namely the center of the smallest enclosing ball of the simplex.

Suppose $P$ is an $\varepsilon$-sample of a smooth surface $S$ with $\varepsilon<0.1$. Recently, it was shown that the intersection points of an $\alpha$-critical simplex with its dual Voronoï cell is either very close to the surface $S$ or very close to the medial axis $M(S)$ [DGRS05]. Therefore, we can classify an $\alpha$-critical simplex as either surface- or medial-axis-critical. The second line of surface reconstruction algorithms that we discussed in the introduction used the $\alpha$-critical simplices for reconstruction. The separation property above is the key reason why these algorithms have been successful. By Lemma 4, we know that in the $\hat{\alpha}$-permutation of $\alpha$ critical simplices, the surface-critical ones all appear before the medial-axis-critical. Therefore, conformal alpha shapes incorporate a local filtering that will allow surface reconstruction based on critical simplices.

\section{Conclusion}

In this paper, we introduce conformal alpha shapes, a variation of the method of alpha shapes, that utilizes a local scale parameter $\hat{\alpha}$ that is invariant to scaling and Euclidean transformations. The local parameter reorders the simplices of the alpha shapes filtration into a new filtration. We show that this filtration has complexes that contain the restricted Delaunay simplices. As such, conformal alpha shapes may be utilized for provable surface reconstruction algorithms that compute candidate sets by filtering. Within the $\hat{\alpha}$-filtration, the critical simplices of the alpha shapes filtration remain critical. Moreover, the new ordering separates the critical simplices that are near the surface from those near the medial axis. Therefore, conformal alpha shapes may also be used by the second type of surface reconstruction algorithms that examine critical simplices. Conformal alpha shapes shed new light on the relationship between the two main approaches in Delaunaybased surface reconstruction algorithms. We hope that this new understanding will allow for a new "universal" theory that unifies the two approaches in the near future.

\section{References}

[AB99] AMENTA N., BERN M.: Surface reconstruction by voronoi filtering. Discrete and Computational Geometry 22 (1999), 481-504.

[ACDl00] Amenta N., Choi S., Dey T. K., Leekha N.: A simple algorithm for homeomorphic surface reconstruction. In 16th Annual ACM Symposium on Computational Geometry (2000), pp. 213-222.

[DGRS05] Dey T. K., Giesen J., RAmos E. A., SAdRi B.: Critical points of the distance to an epsilon-sampling on a surface and flow based surface reconstruction. In 21st Annual ACM Symposium on Computational Geometry (2005). (to appear).

[Ede01] EDELSBRUNNER H.: Geometry and Topology for Mesh Generation. Cambridge University Press, New York, NY, 2001.

[Ede04] EDELSBRUnNer H.: Surface reconstruction by wrapping finite point sets in space. Discrete and Computational Geometry 32 (2004), 231-244.

[EFL98] Edelsbrunner H., Facello M. A., Liang J.: On the definition and the construction of pockets in macromolecules. Discrete Appl. Math. 88 (1998), 83-102.

[EKS83] Edelsbrunner H., Kirkpatrick D. G., Seidel R.: On the shape of a set of points in the plane. IEEE Trans. Inform. Theory IT-29 (1983), 551-559.

[EM94] Edelsbrunner H., MÜCKE E. P.: Threedimensional alpha shapes. ACM Trans. Graphics 13 (1994), 43-72.

[GJ03] Giesen J., John M.: The flow complex: A data structure for geometric modeling. In 14th Annual ACM-SIAM Symposium on Discrete Algorithms (2003), pp. 285-294. 